\title{
Descripción estructural de la formación vegetal subtipo Jubaea chilensis - Lithraea caustica, al interior del Parque Nacional La Campana
}

\author{
Description structural of the vegetal formation subtype Jubaea chilensis - \\ Lithraea caustica within of the La Campana National Park
}

\author{
Rolando H. Díaz Fuentes ${ }^{1 *}$, Alexis Lillo², Alejandro Espinosa ${ }^{3}$, Daniela Pérez Paz ${ }^{3}$
}

\begin{abstract}
RESUMEN
La especie Jubaea chilensis (Molina) Baillon (palma chilena), se distribuye en la zona central de Chile en pequeños remanentes con distinto grado de conservación. El palmar de Ocoa con 62.821 individuos, el mejor conservado de todos, se localiza al interior del Parque Nacional La Campana, en la V Región de Valparaíso, único lugar con resguardo estatal por medio del Sistema Nacional de Áreas Silvestres Protegidas del Estado (SNASPE). No obstante, su hábitat ha sido fuertemente alterado por la alta presión antrópica de comunidades cercanas, quienes históricamente han explotado dicha especie para extraer su savia y sus frutos. El objetivo del presente estudio fue caracterizar la composición florística y estructural, del subtipo Jubaea chilensis - Lithraea caustica, en distintos sectores de densidad media de palma chilena, al interior del Parque Nacional La Campana. Los resultados mostraron la alteración que históricamente ha sufrido dicha especie y su vegetación acompañante, por lo que se puede establecer que el estado de conservación y desarrollo de J. chilensis y, de especies acompañantes, se encuentra estancado o en retroceso, lo que se manifiesta principalmente en la falta de individuos por clase de altura de estípite e individuos de regeneración natural.
\end{abstract}

Palabras clave: palma chilena, estructura vegetal, estados de desarrollo, altura estípite.

\section{ABSTRACT}

The Jubaea chilensis species (chilean palm) is distributed in central Chile in small remnants with varying degrees of conservation. The palmar Ocoa with 62.821 individuals, the best conserved of all, is located within La Campana National Park in the V region of Valparaiso, only place with protected state through the Sistema Nacional de Áreas Silvestres Protegidas del Estado (SNASPE). Nevertheless, their habitat has been heavily altered by high anthropic pressure from near communities who have historically exploited the species, mainly to extract sap. The aim of this study was to characterize the floristic and structural composition of the vegetal subtype Jubaea chilensis - Lithraea caustica, in different sectors of medium density of chilean palm within La Campana National Park. The results showed that the alteration that historically has suffered the species and associated vegetation, being determined that J. chilensis and other species, is stagnant or declining, which manifests mainly a lack of height class individuals stipe and individuals of natural regeneration.

Key words: chilean palm, vegetal structure, development stages, stipe height.

\section{Introducción}

Las palmas o palmeras, se distribuyen mundialmente entre el $44^{\circ}$ norte y $44^{\circ}$ sur, en cuyo contexto geográfico el continente sudamericano es el que alberga una mayor riqueza y diversidad de estas especies, encontrándose aquí cuatro de las cinco subfamilias que existen: Calamoideae, Coryphoideae, Ceroxyloideae y Arecoideae; es decir, 459 especies representadas en 50 géneros

1 Universidad de La Frontera, Facultad de Educación Ciencias Sociales y Humanidades, Departamento de Educación. Temuco, Chile.

2 Universidade Federal de São Paulo, Programa Pós-Graduação Biotecnologia, Campus São José Dos Campos, São Paulo, Brasil.

3 Universidad de La Frontera, Facultad de Ciencias Agropecuarias y Forestales, Departamento de Ciencias Forestales, Temuco, Chile.

* Autor de correspondencia: rolando.diaz@ufrontera.cl

Fecha de Recepción: 10 Agosto, 2016.

Fecha de Aceptación: 23 Febrero, 2017.

DOI: $10.4067 / \mathrm{S} 0718-34292017005000020$ 
(Pintaud et al., 2008). En Chile continental existe la especie endémica Jubaea chilensis (Molina) Baillon (palma chilena), la cual pertenece a la familia Aracaceae; y en Chile insular, específicamente en la isla Robinson Crusoe del Archipiélago de Juan Fernández, la especie Juania australis (Mart.) Drude ex. J. D. Hooker (palmera chonta) perteneciente a la subfamilia Ceroxyloideae, también endémica y no emparentada con J. chilensis (Grau, 2000).

Jubaea chilensis, también denominada kankan por las etnias locales de la zona, es una de las especies más emblemáticas de la flora chilena (Rundel y Cowling, 2013) y, según Parsons (2007), es la más austral de América $\left(30^{\circ} \mathrm{S}\right.$ a $\left.36^{\circ} \mathrm{S}\right)$, siendo su estado de conservación, vulnerable (Benoit, 1989). A pesar de la importancia que posee la especie, ha sufrido una reducción gradual de su población en los últimos 150 años y se estima que en la actualidad solo subsisten alrededor de 120.000 individuos, repartidos en diferentes poblaciones aisladas (González et al., 2009). Según Grau (1996), mediante vestigios arqueológicos, se cree que dicha especie se encontraba también en Isla de Pascua, quizás debido al transporte marítimo de sus semillas desde las costas chilenas. Dicho autor ha encontrado numerosos trozos de endocarpio exactamente iguales a los de J. chilensis a diferentes profundidades y horizontes del suelo en dicha isla, los que muestran perforaciones aparentemente realizadas por algún tipo de roedor. De igual manera, ha encontrado polen fosilizado también muy similar a la especie. Por su parte, la desaparición de esta especie en la Isla de Pascua, estaría dada principalmente por la intervención humana, así como la herbivoría de sus semillas debido a la introducción de la especie Rattus exulans (Mieth y Bork, 2010).

Desde el punto de vista económico J. chilensis, ha sido muy apreciada en la zona central de Chile, debido a sus dos principales productos: su savia, base para la elaboración de miel de palma, y sus semillas (coquitos), quienes son utilizados para la elaboración de dulces. En contraste con su amplio historial de uso, se ha reducido drásticamente la cantidad de individuos de la especie, así como también de su vegetación nativa acompañante, debido principalmente a fuertes alteraciones antrópicas que han reducido su hábitat, matriz fundamental para el establecimiento de la regeneración natural (González et al., 2009). Dichas alteraciones han ocurrido históricamente en microcuencas de la región de Valparaíso, desde mediados de siglo XX (Quintanilla y Morales, 2013), teniendo como factores principales los incendios forestales intencionados y la urbanización (Quintanilla Pérez y Lozano Valencia, 2016).

Como características principales, J. chilensis se desarrolla en la zona central de Chile de manera discontinua, siendo los palmares de Cocalán, con alrededor de 30.000 ejemplares y el sector Ocoa en el interior del Parque Nacional La Campana, localizado en la región de Valparaíso, los fragmentos de mayor importancia, siendo este último, el único lugar protegido, con 62.821 individuos (Michea, 1993).

Esta especie monocotiledónea con características arbóreas, crece en la zona central en tres tipos de clima: mediterráneo marino, mediterráneo subtropical semiárido y mediterráneo templado, llegando a medir hasta 30 metros de altura, alcanzando edades cercanas a los 1.000 años, por lo que es considerada una de las palmeras más longevas del mundo (Grau, 2000).

Aunque J. chilensis se encuentra circunscrita geográficamente dentro del tipo forestal esclerófilo, debido a su fisonomía, constituye una clasificación especial, denominada tipo forestal palma chilena (Donoso, 1981); formación vegetal bosque esclerófilo costero, subtipo Jubaea chilensis Lithraea caustica (palma - litre) (Gajardo, 1994). Aunque es reconocida su importancia tanto ecológica como económica, existen pocos estudios respecto a su caracterización ecológica, aspecto esencial para su conservación. Por consiguiente, el objetivo del presente estudio fue describir la estructura de la formación vegetal subtipo Jubaea chilensis - Lithraea caustica en diferentes sectores de densidad media de palma chilena, al interior del Parque Nacional La Campana.

\section{Método}

Área de estudio. El presente trabajo se realizó al interior del Parque Nacional La Campana, entre los meses de enero a abril del año 2005, específicamente en el cordón montañoso La Campana-El Roble de la Cordillera de la Costa, entre los $32^{\circ} 54^{\prime}$ y $33^{\circ} 55^{\prime}$ latitud sur y los $71^{\circ} 12^{\prime}$ longitud oeste, Chile. Este lugar, localizado en la región de Valparaíso, posee un clima templado cálido con inviernos lluviosos, con aproximadamente $400 \mathrm{~mm}$ promedio de agua al año (Quintanilla y Morales, 2013). La superficie 
que ocupa J. chilensis en el Parque Nacional La Campana, corresponde a 2.763 ha, presentando una distribución irregular, con tres categorías de densidad: alta (113 palmas ha $\left.{ }^{-1}\right)$, media (64 palmas ha-1) y baja (2 palmas ha' $\left.{ }^{-1}\right)$, teniendo en general para toda la población un promedio de 22 palmas ha ${ }^{-1}$, asociándose con especies como: Lithraea caustica (Molina) Hook. et Arn, Quillaja saponaria Molina y Retanilla trinervia (Gillies \& Hook.) Hook. \& Arn., entre otras (Michea, 1988).

Definición de rodales. Para establecer los rodales (sectores) estudiados, se consideró el atributo estructural de densidad media de individuos de palma (64 palmas ha $\left.{ }^{-1}\right)$, puesto que Michea (1988) menciona que en estos lugares J. chilensis se desarrolla a diferentes altitudes y exposiciones, permitiendo verificar la heterogeneidad de su hábitat.

A fin de comparar las distintas situaciones vegetacionales y ecológicas, se escogieron lugares con similar pendiente y altitud, lo que fue corroborado mediante apreciación visual y el uso de cartografía, hipsómetro y GPS. De esta manera, los rodales de estudio quedaron definidos mediante una combinación uniforme de rangos de altitud, pendiente y densidad media de palmas, variando solo la exposición. Para ello, se buscaron lugares donde los elementos mencionados operaran de manera similar; dicho de otra manera, se utilizó lo que Ostle (1965) denomina como bloqueo o control de variables, permitiendo obtener conclusiones respecto de la vegetación y, no tanto, de la topografía. Por consiguiente, los rodales estudiados quedaron conformados de acuerdo a la descripción que se muestra en el Tabla 1.

Descripción de la flora. Se determinó el área mínima para cada rodal, mediante la constancia del 90\% de las especies identificadas (Matteucci y Colma, 1982). Al interior de estas parcelas, se realizó un inventario fitosociológico, determinando sociabilidad (Colinvaux, 1995), forma de vida
(Matteucci y Colma, 1982), cobertura por estrato (Braun-Blanquet, 1950) y abundancia (MOPT, 1992). Para identificar diferencias entre los sectores, se determinó el Índice de Jaccard (Steubing et al., 2002). Cabe señalar que la época en la que fueron tomados los datos correspondió a verano, lo que pudo influir en la cantidad de especies encontradas, especialmente herbáceas.

En un lugar representativo de cada rodal, se confeccionó un perfil horizontal y vertical, con una parcela de $8 \mathrm{~m}$ de ancho y $35 \mathrm{~m}$ de largo, a favor de la pendiente, registrando en su interior, para cada uno de los individuos que tuvieran un diámetro superior a $10 \mathrm{~cm}$, la siguiente información: especie, DAP (diámetro a 1,3 $\mathrm{m}$ de altura), altura del estípite en el caso de las palmas, altura total, altura de comienzo de copa, diámetro de copa de cada individuo y coordenadas $\mathrm{X}$ e $\mathrm{Y}$ de acuerdo a la orientación en la pendiente (Granados y Tapia, 1990). Se consideró también, la identificación de otros elementos importantes como especies pertenecientes a otras formas de vida, como el caso de los cactus y los quintrales y la presencia de individuos de regeneración natural de palma chilena.

Descripción estructural. Se realizó un muestreo estratificado al azar (Prodan et al., 1997), en base a un universo muestral de aproximadamente 65 hectáreas, para lo que se establecieron unidades muestrales rectangulares de $1.000 \mathrm{~m}^{2}$, siguiendo la misma metodología utilizada por Michea (1988). La cantidad de unidades de muestreo se determinó mediante el criterio de intensidad de muestreo (FAO, 1971), realizando un total de cinco parcelas en cada sector, lo que constituyó una intensidad de $3,04 \%$. Al interior de cada unidad de muestreo, se determinó: especie, DAP, altura total y altura de estípite para el caso de las palmas. Paralelamente y con el fin de identificar las especies con mayor grado de ocupación del sitio e importancia de cada una de ellas, se determinó la dominancia relativa,

Tabla 1. Resumen del área de estudio, Parque Nacional La Campana.

\begin{tabular}{cllcccc}
\hline Estratos & \multicolumn{1}{c}{ Sector } & Exposición & $\begin{array}{c}\text { Altitud promedio } \\
(\mathrm{msnm})\end{array}$ & $\begin{array}{c}\text { Pendiente promedio } \\
(\%)\end{array}$ & $\begin{array}{c}\text { Superficie } \\
(\text { ha })\end{array}$ & $\begin{array}{c}\text { Densidad de palmas } \\
\left(\text { palmas ha }^{-1}\right)\end{array}$ \\
\hline 1 & Cerro El Labrado & Oeste & 660 & 52 & 14,5 & 34 \\
2 & Monte El Guanaco & Sur-este & 720 & 75 & 5,64 & 14 \\
3 & Cerro Las Placetas & Norte & 748 & 70 & 35,5 & 14 \\
4 & Cerro El Labrado & Sur-oeste & 677 & 39 & 10,1 & 26 \\
\hline
\end{tabular}


utilizando el grado de ocupación del sitio, por lo que se consideró para ello el área basal.

Considerando que en general, para el caso de las palmas no es posible evaluar su edad mediante el diámetro, para analizar la estructura cuantitativa de la población de J. chilensis, se utilizó la metodología usada por Michea y Valdivia (1987) y Michea (1988), quien correlaciona la altura total (en clases), con la cantidad de individuos por hectárea y sus estados de desarrollo (Regeneración: individuos de hoja simple; Infantil: individuos con primeras hojas compuestas, hasta su finalización en diámetro; Juvenil: desde su crecimiento en altura, hasta antes del comienzo de la fructificación; Adulto: individuos que fructifican) utilizando esta vez la altura de estípite y no la de altura total.

\section{Resultados}

Caracterización de la flora y la estructura de la formación palma chilena. Los sectores: 2 Monte El Guanaco de exposición sur-este, 3 Cerro Las Placetas de exposición norte y 4 Cerro El Labrado de exposición sur-oeste, presentaron igual superficie mínima de expresión vegetal, con $128 \mathrm{~m}^{2}$, conteniendo en promedio 14 especies, mientras que el sector 1 Cerro El Labrado de exposición oeste, presentó una superficie menor, de $64 \mathrm{~m}^{2}$ con un menor número de especies (Tabla 2).

Según el inventario fitosociológico, en los sectores Cerro El Labrado de exposición oeste y Cerro Las Placetas de exposición norte, localizados en lugares mayormente soleados, predominan especies arbustivas y herbáceas, mientras que en lugares más sombríos, sectores Monte El Guanaco de exposición sur-este y Cerro El Labrado de exposición sur-oeste, lo hacen especies arbóreas y arbustivas, destacando la formación de matorral de L. caustica creciendo vegetativamente en cepas de forma achaparrada, junto con algunos individuos de Baccharis sp.

De acuerdo al índice de Jaccard, entre los sectores de exposición oeste y norte y, entre sur-este y sur-oeste son los que comparten una mayor cantidad de flora (40\%), mientras que al comparar lugares con mayor y menor grado de luminosidad y grado de humedad entre sectores de exposición oeste y sur-oeste y entre oeste y sur-este, la cantidad de especies compartidas baja a un $26,3 \%$ y $35,8 \%$ respectivamente; por otro lado el sector de exposición sur-este posee un índice de similitud de 31,6\% con el sector de exposición norte, mientras que este, alcanza un $29,2 \%$ de similitud con el sector sur-oeste. En todos las exposiciones, siempre J. chilensis fue la especie con mayor grado de ocupación de sitio, con un índice de dominancia relativa de $65,3 \%$ para el sector sur-este, un $95,2 \%$ para el sector oeste, un 91,9\% para el Sur-Oeste y un $97,8 \%$ para el sector norte.

De los perfiles correspondientes a los sectores de exposición oeste, sur-este y sur-oeste (Figura 1), es posible observar que J. chilensis, L. caustica, Cryptocarya alba (Molina) Looser y Azara celastrina D. Don, son las especies que más caracterizan estos lugares, exceptuando el sector Cerro Las Placetas de exposición norte, donde dichas especies, a excepción de J. chilensis, casi no se presentaron, siendo reemplazadas en abundancia, principalmente por Colliguaja odorifera Molina y $R$. trinervia; mientras que en lugares un poco más altos apareció Puya chilensis Molina.

Por medio de los perfiles, se puede apreciar la altura alcanzada por J. chilensis, siendo mayores, en lugares relativamente más húmedos y sombríos (sectores sur-este y sur-oeste), misma situación que su vegetación acompañante donde $C$. alba y A. celastrina alcanzan mayores alturas y mejores estados de desarrollo; en tanto la especie $Q$. saponaria solo aparece en el sector sur-este con un mayor desarrollo y presencia, observándose además, la escaza presencia de individuos aislados de regeneración natural de J. chilensis, a diferencia del resto de los estratos, donde prácticamente no se observaron.

En cuanto a la estructura cuantitativa de la vegetación acompañante, los sectores de exposición sur-este (Tabla 4) y sur-oeste (Tabla 6) presentaron similar cantidad de especies arbóreas (seis y siete, respectivamente), encontrándose especies como: L. caustica, A. celastrina, C. alba y $Q$. saponaria. En ambas exposiciones, se observó una distribución diamétrica multietánea, con predomino de árboles que superaron los $20 \mathrm{~cm}$ de diámetro, especialmente de individuos de $Q$. saponaria, los que alcanzan un diámetro superior a los 40 y $42 \mathrm{~cm}$ en cada sector respectivamente, aunque en el sector de exposición sur-este, la densidad de dicha especie se distribuye frecuentemente entre los 10 y los $40 \mathrm{~cm}$, situación distinta al sector de exposición Sur-Oeste que solo se distribuye en 2 clases diamétricas, predominando en área basal, siempre en ambos lugares, la especie J. chilensis. 
Tabla 2. Inventario fitosociológico de cuatro sectores de densidad media de Jubaea chilensis en el Parque Nacional La Campana.

\begin{tabular}{|c|c|c|c|c|}
\hline Especie & Forma de vida & Cobertura por estrato & Sociabilidad & Abundancia \\
\hline \multicolumn{5}{|l|}{ Oeste área mínima: 64 m² } \\
\hline Baccharis sp. & 2 & 1 & 3 & 3 \\
\hline Conanthera bifolia Ruiz et Pav. & 3 & 1 & 1 & 3 \\
\hline Colliguaja odorifera Mol. & 2 & 3 & 3 & 5 \\
\hline Lithraea caustica (Molina) Hook. et Arn. & 2 & 2 & 3 & 3 \\
\hline Azara celastrina D. Don. & 2 & 2 & 3 & 3 \\
\hline Jubaea chilensis (Molina) Baillon & 1 & 2 & 5 & 3 \\
\hline Retanilla trinervia (Gillies et Hook.) Hook. \& Arn. & 2 & 1 & 3 & 4 \\
\hline
\end{tabular}

Sur-este área mínima: $128 \mathrm{~m}^{2}$

Escallonia pulverulenta (Ruiz et Pav.) Pers.

Quillaja saponaria Molina

Peumus boldus Molina

Azara celastrina D. Don

Retanilla trinervia

Chamaemelum mixtum (L.) All.

Lithraea caustica

Jubaea chilensis

Moscharia sp.

Baccharis linearis (Ruiz et Pav.) Pers.

Aristeguietia salvia (Colla) R.M. King et H. Rob

\begin{tabular}{llll}
2 & 1 & 3 & 2 \\
1 & 2 & 3 & 3 \\
1 & 2 & 3 & 4 \\
2 & 2 & 3 & 3 \\
2 & 3 & 3 & 5 \\
3 & 1 & 1 & 2 \\
2 & 3 & 3 & 4 \\
1 & 2 & 5 & 3 \\
3 & 1 & 1 & 2 \\
3 & 1 & 1 & 2 \\
2 & 1 & 1 & 2 \\
\hline
\end{tabular}

Norte área mínima: $128 \mathrm{~m}^{2}$

Colliguaja odorifera

Stipa sp.

Proustia cuneifolia D. Don

Baccharis linearis

Retanilla trinervia

Moscharia sp.

Acacia caven (Molina) Molina

Puya chilensis Molina

Baccharis sp.

Aristeguietia salvia (Colla) R.M. King \& H. Rob

Tristerix aphyllus (Miers ex DC.) Barlow et Wiens

Lithraea caustica

Quillaja saponaria

Jubaea chilensis

\begin{tabular}{llll}
2 & 2 & 3 & 4 \\
3 & 1 & 3 & 3 \\
3 & 1 & 3 & 2 \\
3 & 2 & 3 & 3 \\
2 & 4 & 3 & 5 \\
3 & 1 & 2 & 4 \\
2 & 3 & 3 & 4 \\
3 & 4 & 2 & 5 \\
3 & 1 & 3 & 2 \\
2 & 1 & 3 & 3 \\
4 & 1 & 1 & 2 \\
2 & 2 & 3 & 2 \\
1 & 2 & 3 & 2 \\
1 & 2 & 1 & 2 \\
\hline
\end{tabular}

Sur-oeste área mínima: $128 \mathrm{~m}^{2}$

Chaptalia exscapa (Pers.) Baker var. chilensis (DC.) Burkart

Colliguaja odorifera

Eupatorium salvia

Baccharis linearis

Peumus boldus

Azara celastrina

Lithraea caustica

Jubaea chilensis

Tropaeolum sp.

Stipa sp.

Baccharis marginalis DC

Ephedra andina $\mathrm{K}$. Presl

Echinopsis chiloensis (Colla) Friedrich et Rowley

Tristerix aphyllus

Retanilla trinervia

Sphacele salviae (Lindl). Briq.

Escallonia pulverulenta (Ruiz et Pav.) Pers.

\begin{tabular}{llll}
3 & 1 & 3 & 2 \\
2 & 4 & 3 & 5 \\
2 & 1 & 3 & 4 \\
3 & 2 & 3 & 4 \\
1 & 2 & 3 & 5 \\
2 & 2 & 3 & 4 \\
2 & 2 & 3 & 4 \\
1 & 2 & 1 & 3 \\
3 & 1 & 1 & 3 \\
3 & 1 & 3 & 2 \\
2 & 1 & 3 & 2 \\
2 & 1 & 3 & 2 \\
2 & 1 & 3 & 2 \\
4 & 1 & 1 & 3 \\
2 & 2 & 3 & 5 \\
3 & 1 & 3 & 2 \\
2 & 1 & 3 & 1 \\
\hline
\end{tabular}

Forma de vida: 1 Árbol; 2 Arbusto; 3 Herbácea; 4 Epífita; 5 Liana. Cobertura por estrato: 1 menos del 5\%; 2 entre 5\% y 25\%; 3 entre $25 \%$ y $50 \%$; 4 entre $50 \%$ y 75\%; 5 entre $75 \%$ y 100\%. Sociabilidad: 1 aislada; 2 en grupos; 3 en matas; 4 pequeñas colonias; 5 formaciones puras. Abundancia: 1 Rara; 2 Poco común; 3 Frecuente; 4 Común; 5 Muy común. 


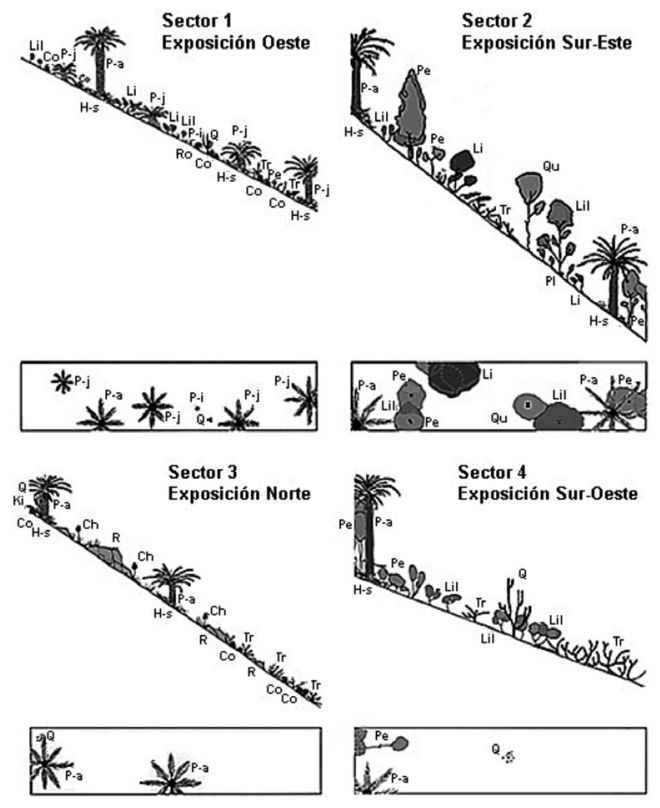

Figura 1. Perfiles vertical y horizontal de Jubaea chilensis en cuatro sectores de densidad media, evaluados en el Parque Nacional La Campana, escala 1:12,5.

P-a: palma adulta; P-i: palma infantil; P-j: palma juvenil; Pl: plántula de palma; H-s: hojas secas de palma; Tr: Retanilla trinervia; Ro: Baccharis linearis; Ki: Tristerix aphyllus; Ch: Puya chilensis; Q: Echinopsis chiloensis. Lil: Azara celestrina; Li: Lithraea caustica; Pe: Cryptocarya alba; Co: Colliguaja odorifera; Qu: Quillaja saponaria; R: roquerío.
Respecto de las exposiciones mayormente soleadas como la oeste (Tabla 3), se pudo observar que fue este el lugar que presentó una mayor cantidad de especies arbóreas, nueve específicamente, las que son L. caustica y Acacia caven (Molina) Molina las que aportan una mayor densidad y área basal, aunque se destaca la presencia de escasos individuos de $Q$. saponaria aportando una buena proporción de área basal. Por otro lado, el sector de exposición norte (Tabla 5), contraponiéndose al resto de los lugares estudiados, se puedo observar que la cantidad de especies acompañantes disminuye ostensiblemente, encontrándose tan solo dos, $L$. caustica y $Q$. saponaria, ambas con poca densidad por clase diamétrica y con poco aporte en área basal.

Por otro lado, las especies L. caustica, $Q$. saponaria y J. chilensis de carácter arbóreas son las que pueden encontrarse en todas las exposiciones, variando en la cantidad de individuos por clase diamétrica. Respecto de otras especies con menores frecuencias y distribución diamétrica, se destaca que en el sector oeste (Tabla 3) es en donde se encontró un mayor número de especies arbóreas, destacando la presencia de Peumus boldus (Molina), Kageneckia oblonga Ruiz y Pav., Schinus latifolius (Gill. ex Lindl.) Engler., A. celastrina y A. caven, siempre con diámetros no superior a $18 \mathrm{~cm}$.

Tabla 3. Tabla de rodal Cerro El Labrado (oeste), Parque Nacional La Campana.

\begin{tabular}{|c|c|c|c|c|c|c|c|c|c|c|c|c|c|c|c|c|c|c|}
\hline \multirow[b]{2}{*}{$\begin{array}{l}C D \\
\mathrm{~cm}\end{array}$} & \multicolumn{2}{|c|}{$\begin{array}{l}\text { Peumus } \\
\text { boldus }\end{array}$} & \multicolumn{2}{|c|}{$\begin{array}{l}\text { Kageneckia } \\
\text { oblonga }\end{array}$} & \multicolumn{2}{|c|}{$\begin{array}{l}\text { Acacia } \\
\text { caven }\end{array}$} & \multicolumn{2}{|c|}{$\begin{array}{l}\text { Lithraea } \\
\text { caustica }\end{array}$} & \multicolumn{2}{|c|}{$\begin{array}{c}\text { Azara } \\
\text { celastrina }\end{array}$} & \multicolumn{2}{|c|}{$\begin{array}{l}\text { Schinus } \\
\text { latifolius }\end{array}$} & \multicolumn{2}{|c|}{$\begin{array}{c}\text { Jubaea } \\
\text { chilensis }\end{array}$} & \multicolumn{2}{|c|}{$\begin{array}{c}\text { Cryptocarya } \\
\text { alba }\end{array}$} & \multicolumn{2}{|c|}{$\underset{\text { saponaria }}{\text { Quillaja }}$} \\
\hline & $\begin{array}{c}N \\
\text { árboles } \\
\text { ha }^{-1}\end{array}$ & $\begin{array}{c}G \\
\mathrm{~m}^{2} \\
\mathrm{ha}^{-1}\end{array}$ & $\begin{array}{c}N \\
\text { árboles } \\
\text { ha }^{-1}\end{array}$ & $\begin{array}{c}G \\
\mathrm{~m}^{2} \\
\mathrm{ha}^{-1}\end{array}$ & $\begin{array}{c}N \\
\text { árboles } \\
\mathrm{ha}^{-1}\end{array}$ & $\begin{array}{c}G \\
\mathrm{~m}^{2} \\
\mathrm{ha}^{-1}\end{array}$ & $\begin{array}{c}N \\
\text { árboles } \\
\mathrm{ha}^{-1}\end{array}$ & $\begin{array}{c}G \\
\mathrm{~m}^{2} \\
\mathrm{ha}^{-1}\end{array}$ & $\begin{array}{c}N \\
\text { árboles } \\
\text { ha }^{-1}\end{array}$ & $\begin{array}{c}G \\
\mathrm{~m}^{2} \\
\mathrm{ha}^{-1}\end{array}$ & $\begin{array}{c}N \\
\text { árboles } \\
\text { ha }^{-1}\end{array}$ & $\begin{array}{c}G \\
\mathrm{~m}^{2} \\
\mathrm{ha}^{-1}\end{array}$ & $\begin{array}{c}N \\
\text { palmas } \\
\mathrm{ha}^{-1}\end{array}$ & $\begin{array}{c}G \\
\mathrm{~m}^{2} \\
\mathrm{ha}^{-1}\end{array}$ & $\begin{array}{c}N \\
\text { árboles } \\
\mathrm{ha}^{-1}\end{array}$ & $\begin{array}{c}G \\
\mathrm{~m}^{2} \\
\mathrm{ha}^{-1}\end{array}$ & $\begin{array}{c}N \\
\text { árboles } \\
\mathrm{ha}^{-1}\end{array}$ & $\begin{array}{c}G \\
\mathrm{~m}^{2} \\
\mathrm{ha}^{-1}\end{array}$ \\
\hline 10 & 2 & 0,02 & 6 & 0,05 & 16 & 0,14 & 8 & 0,07 & 4 & 0,03 & 2 & 0,02 & 2 & 0,02 & 2 & 0,2 & - & - \\
\hline 12 & 2 & 0,03 & - & - & 12 & 0,15 & 12 & 0,15 & 2 & 0,02 & 2 & 0,02 & - & - & 2 & 0,2 & - & - \\
\hline 14 & 2 & 0,03 & - & - & 4 & 0,06 & 14 & 0,22 & - & - & - & - & - & - & - & - & - & - \\
\hline 16 & - & - & - & - & 2 & 0,05 & 6 & 0,12 & - & - & - & - & - & - & 2 & 0,04 & - & - \\
\hline 18 & - & - & - & - & 2 & 0,04 & - & - & - & - & - & - & - & - & - & - & - & - \\
\hline 22 & - & - & - & - & - & - & 2 & 0,08 & - & - & - & - & - & - & 2 & 0,08 & _- & - \\
\hline 26 & - & - & - & - & - & - & - & - & - & - & - & - & 2 & 0,11 & - & - & - & - \\
\hline 40 & - & - & - & - & - & - & - & - & - & - & - & - & 4 & 0,50 & - & - & - & - \\
\hline 42 & - & - & - & - & - & - & - & - & - & - & - & - & - & - & - & - & 2 & 0,28 \\
\hline 60 & - & - & - & _- & _- & - & _ & - & - & - & - & - & 2 & 0,57 & - & - & - & - \\
\hline 64 & - & - & - & - & - & - & - & - & - & - & - & - & 2 & 0,66 & - & - & - & - \\
\hline 68 & - & - & - & - & - & - & - & - & - & - & - & - & 4 & 1,45 & - & - & - & - \\
\hline 72 & - & - & - & - & - & - & - & - & - & - & - & - & 2 & 0,81 & - & - & - & - \\
\hline 74 & - & - & - & - & - & - & - & - & - & - & - & - & 4 & 1,77 & - & - & - & - \\
\hline 80 & - & - & - & - & - & - & - & - & - & - & - & - & 6 & 3,02 & - & - & - & - \\
\hline 82 & - & - & - & - & - & - & - & - & - & - & - & - & 2 & 1,08 & - & - & - & - \\
\hline 84 & - & - & - & - & - & - & - & - & - & - & - & - & 2 & 1,13 & - & - & - & - \\
\hline 92 & - & - & - & - & - & - & - & - & - & - & - & - & 8 & 5,32 & - & - & - & - \\
\hline 94 & - & - & - & - & - & - & - & - & - & - & - & - & 12 & 8,51 & - & - & - & - \\
\hline 96 & - & - & - & - & - & - & - & - & - & - & - & - & 6 & 4,40 & - & - & - & - \\
\hline 100 & - & - & - & - & - & - & - & - & - & - & - & - & 4 & 3,14 & - & - & - & - \\
\hline 104 & - & - & - & - & - & - & - & - & - & - & - & - & 2 & 1,70 & - & - & - & - \\
\hline Total & 6 & 0,07 & 6 & 0,05 & 36 & 0,44 & 42 & 0,64 & 6 & 0,06 & 4 & 0,04 & 64 & 34,19 & 8 & 0,16 & 2 & 0,28 \\
\hline
\end{tabular}


Tabla 4. Tabla de rodal Monte El Guanaco (sur-este), Parque Nacional La Campana.

\begin{tabular}{|c|c|c|c|c|c|c|c|c|c|c|c|c|}
\hline \multirow[b]{2}{*}{$\begin{array}{l}C D \\
\mathrm{~cm}\end{array}$} & \multicolumn{2}{|c|}{$\begin{array}{l}\text { Kageneckia } \\
\text { oblonga }\end{array}$} & \multicolumn{2}{|c|}{$\begin{array}{l}\text { Lithraea } \\
\text { caustica }\end{array}$} & \multicolumn{2}{|c|}{$\begin{array}{c}\text { Azara } \\
\text { celastrina }\end{array}$} & \multicolumn{2}{|c|}{$\begin{array}{c}\text { Jubaea } \\
\text { chilensis }\end{array}$} & \multicolumn{2}{|c|}{$\begin{array}{c}\text { Cryptocarya } \\
\text { alba }\end{array}$} & \multicolumn{2}{|c|}{$\begin{array}{c}\text { Quillaja } \\
\text { saponaria }\end{array}$} \\
\hline & $\begin{array}{c}N \\
\text { árboles } \\
\text { ha }^{-1}\end{array}$ & $\begin{array}{c}G \\
\mathrm{~m}^{2} \\
\mathrm{ha}^{-1}\end{array}$ & $\begin{array}{c}N \\
\text { árboles } \\
\text { ha }^{-1}\end{array}$ & $\begin{array}{c}G \\
\mathrm{~m}^{2} \\
\mathrm{ha}^{-1}\end{array}$ & $\begin{array}{c}N \\
\text { árboles } \\
\text { ha }^{-1}\end{array}$ & $\begin{array}{c}G \\
\mathrm{~m}^{2} \\
\mathrm{ha}^{-1}\end{array}$ & $\begin{array}{c}N \\
\text { palmas } \\
\text { ha }^{-1}\end{array}$ & $\begin{array}{c}G \\
\mathrm{~m}^{2} \\
\mathrm{ha}^{-1}\end{array}$ & $\begin{array}{c}N \\
\text { árboles } \\
\mathrm{ha}^{-1}\end{array}$ & $\begin{array}{c}G \\
\mathrm{~m}^{2} \\
\mathrm{ha}^{-1}\end{array}$ & $\begin{array}{c}N \\
\text { árboles } \\
\text { ha }^{-1}\end{array}$ & $\begin{array}{c}G \\
\mathrm{~m}^{2} \\
\mathrm{ha}^{-1}\end{array}$ \\
\hline 10 & 4 & 0,03 & 44 & 0,39 & 14 & 0,12 & - & - & 26 & 0,23 & 4 & 0,03 \\
\hline 12 & 2 & 0,03 & 48 & 0,58 & 8 & 0,09 & - & - & 34 & 0,40 & 18 & 0,22 \\
\hline 14 & - & - & 34 & 0,54 & 8 & 0,12 & - & - & 14 & 0,22 & 10 & 0,17 \\
\hline 16 & - & - & 6 & 0,13 & - & - & - & - & - & - & 12 & 0,26 \\
\hline 18 & - & - & - & - & 4 & 0,10 & - & - & - & - & 2 & 0,05 \\
\hline 20 & - & - & - & - & - & - & - & - & - & - & 12 & 0,39 \\
\hline 22 & - & - & - & - & - & - & - & - & - & - & 10 & 0,38 \\
\hline 24 & - & - & - & - & - & - & - & - & - & - & 2 & 0,09 \\
\hline 26 & - & - & - & - & - & - & - & - & - & - & 10 & 0,54 \\
\hline 28 & - & - & - & - & - & - & - & - & - & - & 2 & 0,12 \\
\hline 30 & - & - & 2 & 0,14 & - & - & - & - & - & - & 2 & 0,14 \\
\hline 32 & - & - & - & - & - & - & - & - & - & - & 2 & 0,16 \\
\hline 34 & - & - & - & - & - & - & - & - & - & - & 4 & 0,37 \\
\hline 38 & - & - & - & - & - & - & - & - & - & - & 2 & 0,24 \\
\hline 40 & - & - & - & - & - & - & - & - & - & - & 4 & 0,50 \\
\hline 60 & - & - & - & - & - & - & 2 & 0,58 & - & - & - & - \\
\hline 66 & - & - & - & - & - & - & 2 & 0,71 & - & - & - & - \\
\hline 78 & - & - & - & - & - & - & 2 & 0,98 & - & - & - & - \\
\hline 80 & - & - & - & - & - & - & 2 & 1,03 & - & - & - & - \\
\hline 82 & - & - & - & - & - & - & 2 & 1,08 & - & - & - & - \\
\hline 84 & - & - & - & - & - & - & 2 & 1,11 & - & - & - & - \\
\hline 86 & - & - & - & - & - & - & 2 & 1,16 & - & - & - & - \\
\hline 88 & - & - & - & - & - & - & 2 & 1,20 & - & - & - & - \\
\hline 92 & - & - & - & - & - & - & 2 & 1,33 & - & - & - & - \\
\hline 94 & - & - & - & - & - & - & 2 & 1,42 & - & - & - & - \\
\hline 118 & - & - & - & - & - & - & 2 & 2,19 & - & - & - & - \\
\hline Total & 6 & 0,06 & 134 & 1,78 & 34 & 0,44 & 22 & 12,79 & 74 & 0,85 & 96 & 3,68 \\
\hline
\end{tabular}

Tabla 5. Tabla de rodal Cerro Las Placetas (norte), Parque Nacional La Campana.

\begin{tabular}{|c|c|c|c|c|c|c|}
\hline \multirow[b]{2}{*}{$\begin{array}{l}C D \\
\mathrm{~cm}\end{array}$} & \multicolumn{2}{|c|}{$\begin{array}{l}\text { Lithraea } \\
\text { caustica }\end{array}$} & \multicolumn{2}{|c|}{$\begin{array}{c}\text { Jubaea } \\
\text { chilensis }\end{array}$} & \multicolumn{2}{|c|}{$\begin{array}{c}\text { Quillaja } \\
\text { saponaria }\end{array}$} \\
\hline & $\begin{array}{c}N \\
\text { árboles } \\
\text { ha }^{-1}\end{array}$ & $\begin{array}{c}G \\
\mathrm{~m}^{2} \\
\mathrm{ha}^{-1}\end{array}$ & $\begin{array}{c}N \\
\text { palmas } \\
\text { ha }^{-1}\end{array}$ & $\begin{array}{c}G \\
\mathrm{~m}^{2} \\
\mathrm{ha}^{-1}\end{array}$ & $\begin{array}{c}N \\
\text { árboles } \\
\text { ha }^{-1}\end{array}$ & $\begin{array}{c}G \\
\mathrm{~m}^{2} \\
\mathrm{ha}^{-1}\end{array}$ \\
\hline 10 & 2 & 0,02 & - & - & 2 & 0,2 \\
\hline 12 & - & - & - & - & 2 & 0,2 \\
\hline 26 & - & - & 2 & 0,11 & - & - \\
\hline 32 & - & - & 2 & 0,17 & - & - \\
\hline 46 & - & - & - & - & 2 & 0,35 \\
\hline 72 & - & - & 2 & 0,84 & - & - \\
\hline 74 & - & - & 2 & 0,88 & - & - \\
\hline 82 & - & - & 2 & 1,08 & - & - \\
\hline 84 & - & - & 2 & 1,11 & - & - \\
\hline 86 & - & - & 2 & 1,19 & - & - \\
\hline 90 & - & - & 4 & 2,57 & - & - \\
\hline 92 & - & - & 4 & 2,66 & - & - \\
\hline 94 & - & - & 2 & 1,42 & - & - \\
\hline 96 & - & - & 2 & 1,48 & - & - \\
\hline 98 & - & - & 4 & 3,08 & - & - \\
\hline 104 & - & - & 2 & 1,70 & - & - \\
\hline Total & 2 & 0,02 & 32 & 18,29 & 6 & 0,39 \\
\hline
\end{tabular}

Estructura cuantitativa de Jubaea chilensis. Se esperaba encontrar en los diferentes sectores estudiados una distribución multietánea de un monte alto irregular en edad y su representación por estados de desarrollo, los cuatro sectores demostraron una ausencia total de individuos en algunas clases de altura de estípite. Esto se demuestra al revisar los gráficos por sector (Figura 2), donde es posible verificar el evidente estado de degradación en la estructura etaria de la especie. Esto es posible de comprobar, además, al verificar la carencia de individuos en la mayoría de las clases de altura, observándose una distribución casi regular a lo largo de las diferentes clases, con pocos o nula cantidad de individuos, especialmente en los sectores de exposición norte, sur-este y sur-oeste. Una situación preocupante es la limitada o nula cantidad de regeneración natural a excepción del sector de exposición sur-este que al parecer presenta condiciones más favorables para el establecimiento de la regeneración natural. 
Tabla 6. Tabla de rodal Cerro El Labrado (sur-oeste), Parque Nacional La Campana.

\begin{tabular}{|c|c|c|c|c|c|c|c|c|c|c|c|c|c|c|}
\hline \multirow[b]{2}{*}{$\begin{array}{l}C D \\
\mathrm{~cm}\end{array}$} & \multicolumn{2}{|c|}{$\begin{array}{c}\text { Peuтиs } \\
\text { boldus }\end{array}$} & \multicolumn{2}{|c|}{$\begin{array}{l}\text { Lithraea } \\
\text { caustica }\end{array}$} & \multicolumn{2}{|c|}{$\begin{array}{c}\text { Azara } \\
\text { celastrina }\end{array}$} & \multicolumn{2}{|c|}{$\begin{array}{l}\text { Schinus } \\
\text { latifolius }\end{array}$} & \multicolumn{2}{|c|}{$\begin{array}{c}\text { Jubaea } \\
\text { chilensis }\end{array}$} & \multicolumn{2}{|c|}{$\begin{array}{c}\text { Cryptocarya } \\
\text { alba }\end{array}$} & \multicolumn{2}{|c|}{$\begin{array}{c}\text { Quillaja } \\
\text { saponaria }\end{array}$} \\
\hline & $\begin{array}{c}N \\
\text { árboles } \\
\text { ha }^{-1}\end{array}$ & $\begin{array}{c}G \\
\mathrm{~m}^{2} \\
\mathrm{ha}^{-1}\end{array}$ & $\begin{array}{c}N \\
\text { árboles } \\
\mathrm{ha}^{-1}\end{array}$ & $\begin{array}{c}G \\
\mathrm{~m}^{2} \\
\mathrm{ha}^{-1}\end{array}$ & $\begin{array}{c}N \\
\text { árboles } \\
\mathrm{ha}^{-1}\end{array}$ & $\begin{array}{c}G \\
\mathrm{~m}^{2} \\
\mathrm{ha}^{-1}\end{array}$ & $\begin{array}{c}N \\
\text { árboles } \\
\mathrm{ha}^{-1}\end{array}$ & $\begin{array}{c}G \\
\mathrm{~m}^{2} \\
\mathrm{ha}^{-1}\end{array}$ & $\begin{array}{c}N \\
\text { palmas } \\
\text { ha }^{-1}\end{array}$ & $\begin{array}{c}G \\
\mathrm{~m}^{2} \\
\mathrm{ha}^{-1}\end{array}$ & $\begin{array}{c}N \\
\text { árboles } \\
\text { ha }^{-1}\end{array}$ & $\begin{array}{c}G \\
\mathrm{~m}^{2} \\
\mathrm{ha}^{-1}\end{array}$ & $\begin{array}{c}N \\
\text { árboles } \\
\text { ha }^{-1}\end{array}$ & $\begin{array}{c}G \\
\mathrm{~m}^{2} \\
\mathrm{ha}^{-1}\end{array}$ \\
\hline 10 & - & - & 10 & 0,09 & 8 & 0,07 & 4 & 0,04 & - & - & 48 & 0,42 & - & - \\
\hline 12 & 4 & 0,05 & 12 & 0,14 & 8 & 0,10 & 2 & 0,03 & 2 & 0,02 & 20 & 0,24 & - & - \\
\hline 14 & 2 & 0,04 & 4 & 0,06 & 2 & 0,03 & 2 & 0,03 & - & - & 12 & 0,20 & - & - \\
\hline 16 & - & - & - & - & - & - & - & - & 2 & 0,05 & 4 & 0,08 & - & - \\
\hline 18 & - & - & - & - & - & - & - & - & - & - & 2 & 0,05 & - & - \\
\hline 20 & 2 & 0,07 & - & - & - & - & - & - & 2 & 0,06 & 2 & 0,06 & 2 & 0,06 \\
\hline 22 & - & - & - & - & - & - & - & - & 2 & 0,08 & - & - & - & - \\
\hline 30 & - & - & - & - & - & - & - & - & 2 & 0,14 & - & - & - & - \\
\hline 42 & - & - & - & - & - & - & - & - & - & - & - & - & 2 & 0,29 \\
\hline 70 & - & - & - & - & - & - & - & - & 2 & 0,79 & - & - & - & - \\
\hline 72 & - & - & - & - & - & - & - & - & 2 & 0,81 & - & - & - & - \\
\hline 82 & - & - & - & - & - & - & - & - & 2 & 1,06 & - & - & - & - \\
\hline 84 & - & - & - & - & - & - & - & - & 8 & 4,49 & - & - & - & - \\
\hline 88 & - & - & - & - & - & - & - & - & 2 & 1,22 & - & - & - & - \\
\hline 90 & - & - & - & - & - & - & - & - & 2 & 1,39 & - & - & - & - \\
\hline 92 & - & - & - & - & - & - & - & - & 4 & 2,69 & - & - & - & - \\
\hline 96 & - & - & - & - & - & - & - & - & 4 & 2,90 & - & - & - & - \\
\hline 98 & - & - & - & - & - & - & - & - & 2 & 1,51 & - & - & - & - \\
\hline 100 & - & - & - & - & - & - & - & - & 2 & 1,60 & - & - & - & - \\
\hline 102 & - & - & - & - & - & - & - & - & 2 & 1,63 & - & - & - & - \\
\hline 104 & - & - & - & - & - & - & - & - & 2 & 1,73 & - & - & - & - \\
\hline 114 & - & - & - & - & - & - & - & - & 2 & 2,04 & - & - & - & - \\
\hline Total & 8 & 0,15 & 26 & 0,29 & 18 & 0,19 & 8 & 0,10 & 46 & 24,12 & 88 & 1,05 & 4 & 0,35 \\
\hline
\end{tabular}
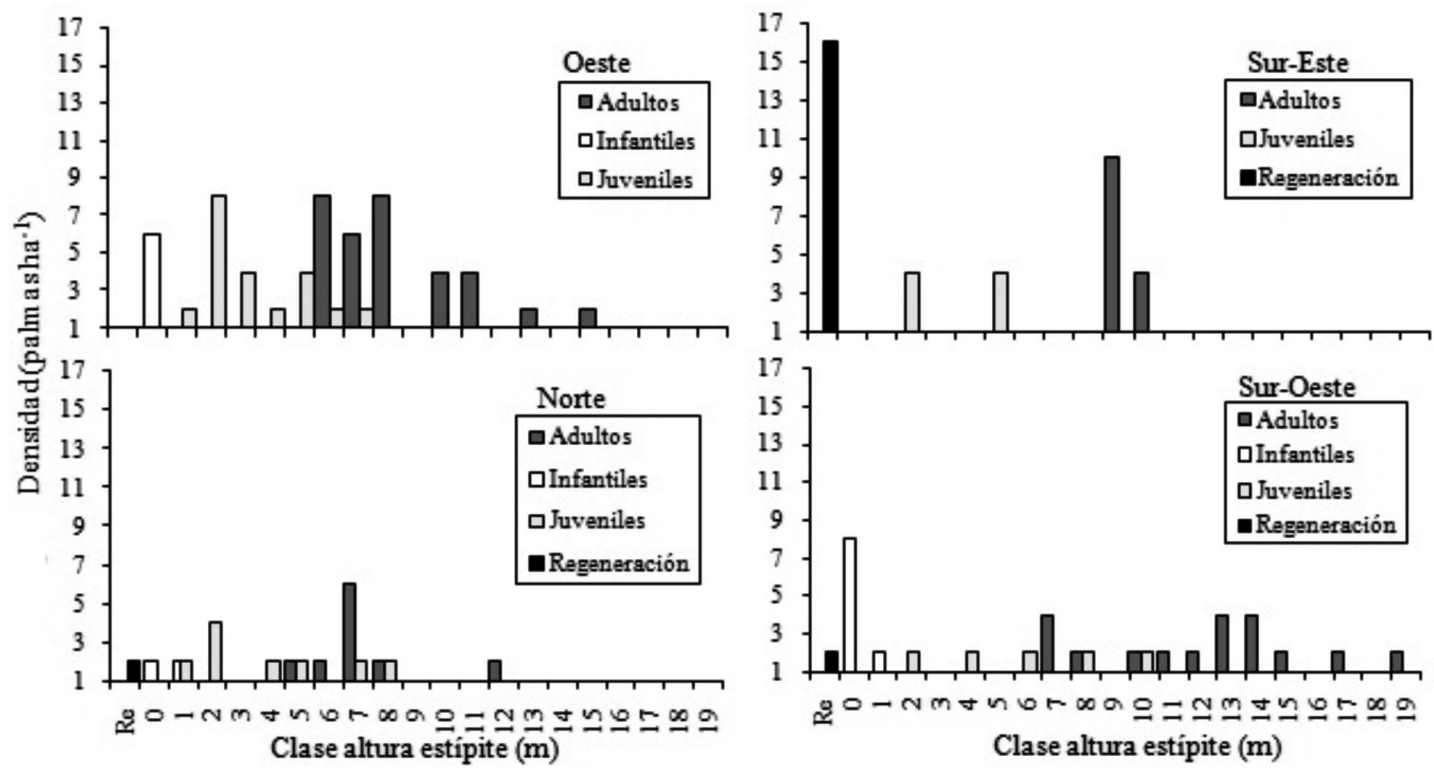

Figura 2. Gráfico clase de altura de estípite de Jubaea chilensis en cuatro sectores de densidad media en el Parque Nacional La Campana. 
En cuanto a individuos adultos, (palmas con potencial de producción de coquitos), se pudo corroborar que el sector de exposición oeste presenta la mayor cantidad de estos individuos, 34 palmas ha ${ }^{-1}$, seguido del sector sur-oeste con 26 palmas ha ${ }^{-1}$, mientras que los sectores sur-este y norte presentaron un total de 14 palmas ha- $^{-1}$. En cuanto a los individuos juveniles, la mayor cantidad fue encontrada en el sector de exposición oeste con 24 palmas ha-1, seguido de los sectores de exposición norte, sur-oeste y sur-este con 14,10 y 8 palmas ha ${ }^{-1}$ respectivamente, los individuos infantiles en tanto, presentaron una densidad de 10 y 4 palmas ha ${ }^{-1}$ en los sectores sur-oeste y norte respectivamente, mientras que para los sectores oeste y sur-este no se presentaron individuos en esta categoría de desarrollo.

Es importante destacar la ausencia de regeneración natural de J. chilensis, la que se presenta de manera muy poco frecuente, especialmente en el sector Cerro El Labrado de exposición oeste donde no se pudo encontrar plántulas de hoja simple, mientras que en el resto de los sectores solo se encontraron algunos individuos, teniendo para los sectores Cerro Las Placetas y El Labrado de exposiciones norte y suroeste respectivamente, un total de 2 plántulas ha ${ }^{-1}$, mientras que en el sector de exposición sur-este, se encontró la mayor cantidad, correspondiendo a un total de 16 plántulas ha ${ }^{-1}$.

\section{Discusión}

La composición de la flora y la estructura de la comunidad vegetal de palma chilena en los sectores Cerro el Labrado de exposición oeste, Monte El Guanaco de exposición sur-este y Cerro El Labrado de exposición sur-oeste, se encontró la mayor abundancia de las especies J. chilensis y L. caustica, debido a que presentaría las mejores condiciones microclimáticas según su autoecología. El caso contrario lo constituye el sector Cerro Las Placetas de exposición norte, el que se presenta con una menor cantidad de especies arbóreas y menor estructura cuantitativa, lo que puede deberse fundamentalmente a las inclemencias edafoclimáticas, asociadas fundamentalmente con las altas temperaturas alcanzadas durante el día, más la erosión del suelo debido a su desprotección y menor cantidad de hojarasca. Por ello es que predominan en abundancia, principalmente en sectores más bajos, especies como $R$. trinervia o $C$. odorífera y, en lugares más altos lo hace $P$. chilensis, estando estas tres especies mejor adaptadas a este tipo de ambiente. De igual modo, destaca la presencia, aunque no de forma abundante, la especie Echinopsis chiloensis (Colla) Friedr. et Rowl. (quisco) en los sectores de exposición oeste, norte y sur-oeste, especialmente por ser lugares mayormente soleados, mientras que un mayor desarrollo en altura de esta especie es logrado en lugares más húmedos cercano al ecotono, sectores con mayor cantidad de materia orgánica y, por lo tanto, con mayores aportes nutricionales, los que son producidos por la descomposición de la hojarasca. Se debe considerar para efecto de la composición florística que el área mínima en cada uno de los sectores podría ser mayor y contener un número mayor de especies especialmente herbáceas, quienes son depredadas con mayor facilidad por el ganado doméstico presente comúnmente en el interior del parque.

Según Flores-Toro y Aguirre-Saavedra (2008), las especies anteriormente señaladas y, presentadas con mayor frecuencia dentro de los sectores estudiados, serían constituyentes inequívocos del tipo forestal palma chilena y es una situación comparable a la del palmar El Salto, localizado en la comuna de Viña del Mar, estando diferenciados por el número de especies presentes, 545 para el Parque Nacional La Campana y 220 para el palmar El Salto, este último con menor superficie y un mayor grado de alteración antrópica.

En cuanto a la caracterización estructural de la formación dominada por J. chilensis, es posible contrastar que una mayor estructura o protección del dosel superior, mejora las condiciones para el establecimiento de la regeneración natural de ésta especie. Ello se observa de mejor forma en los sectores de exposición sur-este y en menor grado sur-oeste, lugares donde las condiciones edafológicas mejoran principalmente por el aporte de hojarasca de la especie L. caustica, y otras especies con mayor desarrollo en altura los que crean las condiciones más favorables para el establecimiento de las plántulas y su desarrollo según lo demandado por su autoecología.

Desde el punto de vista de la estructura cuantitativa de J. chilensis, se deduce que esta, en ausencia de un ambiente adecuado se desarrolla poco en altura, es decir, se mantiene de forma achaparrada hasta que las condiciones del medio 
puedan mejorar para crecer más rápidamente en altura, hecho principalmente observable en el sector Cerro Las Placetas de exposición norte y Cerro El Labrado de exposición oeste, donde se identificó una mayor cantidad de individuos adultos relativamente más pequeños en proporción al resto de los estados de desarrollo; en comparación por ejemplo, con el sector Monte El Guanaco de exposición sur-este, donde el diámetro de esta especie, se presenta de forma proporcional al tamaño de altura de estípite; esto permite formular la hipótesis, que investigar el desarrollo de J. chilensis mediante clases de altura, ya sea total o de estípite, puede al igual que el diámetro, no ser un buen indicador para conocer fehacientemente su estructura etaria, aconsejándose para futuros estudios, el desarrollo de nuevas técnicas.

Es posible agregar también que, la disminución en el número de individuos de J. chilensis presentada por las distintas clases de altura de estípite, demuestra la alteración ecológica de los sectores estudiados, escaseando individuos juveniles que de acuerdo al historial de manejo, han sido los más demandados para la extracción de savia (González et al., 2009; Wara et al., 2006). Sin embargo, dicha falta de individuos es también apreciada en los individuos infantiles, ya que en el sector Monte El Guanaco de exposición sur-este su densidad es nula, lo que da una idea acerca del tiempo de exposición a la alteración ecológica que ha transcurrido en los lugares investigados.

Para el caso de la regeneración natural, las especies acompañantes, especialmente arbustivas, debido principalmente a su aporte en hojarasca $y$, por ende, una mayor cantidad de materia orgánica, podrían mejorar las condiciones para el establecimiento de la regeneración natural; sin embargo, dichas especies se encuentran también con fuertes grados de alteración, principalmente debido al ramoneo y tránsito de animales vacunos, las que no lograrían mejorar las condiciones del micrositio y por tanto el desarrollo de la especie, especialmente para los sectores con mayores temperaturas, donde no existe una protección importante desde un estrato superior, lugares con exposición fundamentalmente norte. En estos lugares, especialmente en el sector de exposición oeste, lugar con la mayor densidad de palmas adultas (productoras de frutos), es donde la regeneración natural no se ha podido establecer de forma satisfactoria, situación que hace suponer que el factor ambiental, más el factor antrópico de extracción de semillas, es uno de los más determinantes a la hora de establecer las pequeñas plántulas. Ahora, desde el punto de vista antrópico, se suma la presencia de ganado doméstico, especialmente bovinos, que se alimentan de la regeneración y de los frutos de J. chilensis, más la extracción indiscriminada de frutos y tierra de hoja, situación menos habitual en sectores de uso público y de educación ambiental, los que junto a factores naturales como la herbívora principalmente de roedores como Octodon degus (Yates et al., 1994) y lagomorfos introducidos, hacen poco viable el establecimiento de la regeneración natural, manteniendo los sectores investigados en este trabajo, en un estado crítico de degradación vegetal.

En cuanto a la menor frecuencia encontrada en los tipos de desarrollo según individuos de regeneración natural, infantiles y juveniles de J. chilensis, se demuestra que, por lo menos los sectores de densidad media investigados se encuentran con un alto grado de la alteración, puesto que al comparar los resultados determinado por Michea y Valdivia (1987) (un monte alto irregular), hasta febrero de 2005, fecha en el que se obtuvieron los datos del presente estudio, solo se ha constatado la continua pérdida de individuos por clases etarias, visualizándose un envejecimiento de la comunidad vegetal de palma chilena.

Este estudio demuestra que al comparar estudios anteriores con nuestros resultados, es posible inferir la inexistencia de acciones adecuadas para la recuperación de la estructura ecológica de palma chilena. Esto permite dar cuenta del estado de vulnerabilidad que se encuentra ésta especie en los lugares estudiados, causado principalmente por acción antrópica, donde fue posible observar compactación del suelo, destrucción de la vegetación, presencia de ganado doméstico (vacuno) y extracción sin control ni autorización de frutos de palma.

\section{Agradecimientos}

Los autores agradecen a la Corporación Nacional Forestal (CONAF), Región de Valparaíso y la U.G. Patrimonio Silvestre, por los aportes financieros y logísticos que hicieron posible la presente publicación. Se agradece a Leonardo Moder, Yenny Prieto, e Iván Wagman y al resto del Cuerpo de Guardaparque del Parque Nacional La Campana, sector Ocoa, por la desinteresada colaboración prestada al presente estudio. 


\section{Literatura Citada}

Benoit, I.L.

1989. Libro Rojo de la Flora Terrestre de Chile (Primera Parte). Corporación Nacional Forestal (CONAF). Santiago, Chile, $157 \mathrm{p}$.

Braun-Blanquet, J.

1950. Sociología Vegetal. Estudios de las Comunidades Vegetales. Acme Agency. Buenos Aires, Argentina, 444 p.

Colinvaux, $\mathrm{P}$.

1995. Introducción a la ecología. Limusa. México D.F., México, 679 p.

Donoso, $\mathrm{C}$.

1981. Tipos forestales de los bosques nativos de Chile. FAOCONAF, (Documento de trabajo N 38). Santiago, Chile, 70 p. FAO

1971. Planificación de un inventario forestal. Dirección de Recursos Forestales, Departamento de Montes, Organización de las Naciones Unidas para la Agricultura y la Alimentación. Roma, Italia, 173 p.

Flores-Toro, L.; Aguirre-Saavedra, F.

2008. Riqueza florística del Santuario de la Naturaleza Palmar El Salto, comuna de Viña del Mar, Región de Valparaíso, Chile. Gayana Botánica, 65 (1): 1-13.

Gajardo, R.

1994. La vegetación natural de Chile. Clasificación y distribución geográfica. Universitaria. Santiago, Chile, $165 \mathrm{p}$.

González, L.A.; Bustamante, R.O.; Navarro, R.M.; Herrera, M.A.; Ibáñez, M.T.

2009. Ecology and Management of the Chilean Palm (Jubaea chilensis): History, Current Situation and Perspectives. Palms, 53 (2): 68-74.

Granados, D.; Tapia, R.

1990. Comunidades vegetales. Colección Cuadernos Universitarios. Serie Agronomía No 19. Universidad Autónoma de Chapingo. Chapingo, México, 235 p.

Grau, J.

1996. Jubaea, the Palm of Chile and Easter Island? Rapa Nui Journal, 10 (2): 37-40.

Grau, J.

2000. Palmeras. Oikos. Santiago, Chile, 337 p.

Matteucci, S.D.; Colma, A.

1982. Metodología para el estudio de la vegetación. Secretaría General de la Organización de los Estados Americanos. Washington, D.C., US, 168 p.

Michea, G.

1988. Estudio poblacional de Palma Chilena (Jubaea chilensis) en el sector Ocoa, Parque Nacional La Campana. Revista Medio Ambiente, 9 (1): 124-130.

Michea, G.; Valdivia, A.

1987. Inventario forestal de Palma Chilena Jubaea chilensis en el sector Ocoa, Parque Nacional La Campana. Corporación Nacional Forestal, (Informe Técnico). Santiago, Chile, 46 p.
Michea, G.

1993. Las palmeras chilenas: Historia de sobrevivencia. Revista Chile Forestal, 204: 32-33.

Mieth, A.; Bork, H.R.

2010. Humans, climate or introduced rats - which is to blame for the woodland destruction on prehistoric Rapa Nui (Easter Island)? J. Archaeol Sci, 37 (2): 417-426.

MOPT (Ministerio de Obras Públicas y Transportes)

1992. Guía para la elaboración de estudios del medio físico; Contenido y metodología. Ministerio de Obras Públicas y Transportes. Madrid, España, 808 p.

Ostle, B.

1965. Estadística aplicada. Técnicas de la estadística moderna, cuando y donde aplicarlas. Limusa. México D.F., México, $659 \mathrm{p}$.

Parsons, R.F.

2007. The southernmost limits for palms. New Zealand Journal of Botany, 45 (3): 477-478.

Pintaud, J.C.; Galeano, G.; Balslev, H.; Bernal, R.; Borchsenius,

F.; Ferreira, E.

2008. Las palmeras de América del Sur: diversidad, distribución e historia evolutiva. Revista Peruana de Biología, 15 (1): 7-29.

Prodan, M.; Peters, R.; Cox,F.; Real; P.

1997. Mensura Forestal. Instituto Interamericano de Cooperación para la Agricultura (IICA)/BMZ/GTZ. San José, Costa Rica, 561 p.

Quintanilla Pérez, V.; Lozano Valencia, P.

2016. Valoración biogeográfica del bosque mediterráneo esclerófilo con palmeras (Jubaea chilensis Mol (Baillon)) en la cuenca del Quiteño (Chile), a partir de la aplicación del método de valoración Lanbioeva. Pirineos, 171: e018.

Quintanilla, V.; Morales, M.

2013. Perturbaciones de los fuegos de verano en la palma más austral del mundo (Jubaea chilensis (mol.) Baillon) en microcuencas costeras de la Zona Mediterránea de Chile. Cuadernos Geográficos, 52 (1): 129-152.

Rundel, P.W.; Cowling, R.M.

2013. Mediterranean-Climate Ecosystems. In Levin SA eds. Encyclopedia of Biodiversity Waltham. Academic Press. Waltham, US, pp. 212-222.

Steubing L., Godoy,R.; Alberdi, M.

2002. Métodos de ecología vegetal. Editorial Universitaria S.A. Santiago, Chile. 345 p.

Wara, M.; Bustamante, R.O.; Vázquez, R.A.

2006. Efectos de la herbivoría, el microhábitat y el tamaño de las semillas en la sobrevivencia y crecimiento de plántulas de la Palma Chilena. Ambiente y Desarrollo, 22 (2): 55-62.

Yates, L.R.; Saiz,F.; Zunino, S.

1994. Octodon degus: Valor nutricional y preferencia del recurso trófico en el Palmar de Ocoa, Parque Nacional La Campana. Revista Chilena de Historia Natural, 67: 89-99. 
